\title{
IDEAL-IQ in an oncologic population: meeting the challenge of concomitant liver fat and liver iron
}

Sarah Eskreis-Winkler ${ }^{1}$, Giuseppe Corrias ${ }^{1,2}$, Serena Monti ${ }^{4}$, Junting Zheng ${ }^{3}$, Marinela Capanu ${ }^{3}$, Simone Krebs ${ }^{1}$, Maggie Fung ${ }^{5}$, Scott Reeder ${ }^{6}$ and Lorenzo Mannelli ${ }^{1,7^{*}}$ (i)

\begin{abstract}
Background: Cancer patients often have a history of chemotherapy, putting them at increased risk of liver toxicity and pancytopenia, leading to elevated liver fat and elevated liver iron respectively. T1-in-and-out-of-phase, the conventional MR technique for liver fat assessment, fails to detect elevated liver fat in the presence of concomitantly elevated liver iron. IDEAL-IQ is a more recently introduced MR fat quantification method that corrects for multiple confounding factors, including elevated liver iron.

Methods: This retrospective study was approved by the institutional review board with a waiver for informed consent. We reviewed the MRI studies of 50 cancer patients (30 males, 20 females, 50-78 years old) whose exams included (1) T1-in-and-out-of-phase, (2) IDEAL-IQ, and (3) T2* mapping. Two readers independently assessed fat and iron content from conventional and IDEAL-IQ MR methods. Intraclass correlation coefficient (ICC) was estimated to evaluate agreement between conventional MRI and IDEAL-IQ in measuring R2* level (a surrogate for iron level), and in measuring fat level. Agreement between the two readers was also assessed. Wilcoxon signed rank test was employed to compare iron level and fat fraction between conventional MRI and IDEAL-IQ.

Results: Twenty percent of patients had both elevated liver iron and moderate/severe hepatic steatosis. Across all patients, there was high agreement between readers for IDEAL-IQ fat fraction $(I C C=0.957)$ and IDEAL R2* $(I C C=0$. 971) measurements, but lower agreement for conventional fat fraction measurements (ICC $=0.626$ ). The fat fractions calculated with IOP were statistically significantly different from those calculated with IDEAL-IQ (reader 1: $p<0.001$, reader 2: $p<0.001$ ).

Conclusion: Fat measurements using IDEAL-IQ and IOP diverged in patients with concomitantly elevated liver fat and liver iron. Given prior work validating IDEAL-IQ, these diverging measurements indicate that IOP is inadequate to screen for hepatic steatosis in our cancer population.
\end{abstract}

Keywords: Fat fraction, Liver, PDFF, IDEAL-IQ, Chemotherapy, Oncologic imaging

\section{Background}

Patients at tertiary care cancer centers often suffer from disseminated disease and are treated with chemotherapy regimens [1-3]. Chemotherapy can lead to a wide range of adverse effects, including hepatoxicity and pancytopenia. An early sign of hepatotoxicity is hepatic steatosis,

\footnotetext{
* Correspondence: mannellilorenzo@yahoo.it

${ }^{1}$ Department of Radiology, Memorial Sloan Kettering Cancer Center, 1275 York Avenue, New York, NY 10065, USA

7300 East 66th Street, New York, NY 10021, USA

Full list of author information is available at the end of the article
}

which can be recognized on medical imaging studies by indirect signs of the accumulation of fat globules within hepatocytes $[4,5]$. Detecting hepatic steatosis is clinically important, as it often triggers changes in a patient's medical and/or surgical treatment plans [6,7].

Chemotherapy patients are also at risk for pancytopenia, which occurs as a result of chemotherapy-induced bone marrow failure. This often necessitates repeat blood transfusions, which can cause elevated liver iron levels. As such, this patient population may have a higher incidence of concomitantly elevated liver fat and

(C) The Author(s). 2018 Open Access This article is distributed under the terms of the Creative Commons Attribution 4.0 International License (http://creativecommons.org/licenses/by/4.0/), which permits unrestricted use, distribution, and 
elevated liver iron. It is therefore particularly important that the Magnetic Resonance Imaging (MRI) methods used for liver fat detection in this patient population not be confounded by elevated liver iron [8-11].

T1-in-and-out-of-phase (IOP) MRI is the conventional MR method for determining hepatic steatosis, and is included in all abdominal MR protocols at most institutions. In the IOP method, an initial "out-of-phase" (OP) set of images is acquired when there is destructive inference of the water and fat signals, and a subsequent "in phase" (IP) set of is acquired when there is constructive interference of the water and fat signals. The OP and IP images are then used to qualitatively measure liver fat fraction using the following eq. (SI = signal intensity) [12]:

$$
\begin{aligned}
& \text { Liver fat fraction } \%= \\
& \frac{\frac{\text { SI InPhase }_{\text {liver }}}{\text { SI InPhase }_{\text {spleen }}}-\frac{\text { SI OutofPhase }_{\text {liver }}}{\text { SI OutofPhase }_{\text {spleen }}}}{2 * \frac{\text { SI InPhase }_{\text {liver }}}{\text { SI InPhase }_{\text {spleen }}}}
\end{aligned}
$$

However, in patients containing both elevated liver iron and elevated liver fat, fat-induced and iron-induced signal changes cancel, leading to underestimation of both liver fat and liver iron content $[11,13]$. In our patient population, where there may be a high incidence of concomitant elevated liver iron and elevated liver fat, a more robust MR method is needed [14].

Iterative Decomposition of water and fat with Echo Asymmetry and Least squares estimation (IDEAL-IQ) is a more sophisticated chemical-shift encoded fat quantification approach that corrects for several confounding factors, including $\mathrm{T} 1$ bias, eddy currents, noise bias, and T2* effects $[9,15,16]$. In IDEAL-IQ, images are acquired at multiple echo times, and an iterative least-squares decomposition algorithm is employed to simultaneous solve for a fat fraction map, a water fraction map, and an R2* map. By incorporating an R2* map into the algorithm, IDEAL-IQ accounts for T2* effects/field inhomogeneity, and yields a proton density fat fraction (PDFF) not confounded by iron overload [17-21]. IDEAL-IQ also outputs an R2* map, which can be used to identify the presence of iron overload. IDEAL-IQ has been validated in phantoms, in animal models, and in patients, with pathological gold standards [22-27]. Specifically, it has been shown to be highly reliable in patients with iron overload.

However, the utility of IDEAL-IQ has not yet been assessed in an oncologic population. At cancer centers, we expect higher rates of patients with concomitantly elevated liver fat and liver iron. And so, in this paper, we assess the utility of IDEAL-IQ by evaluating to what extent IDEAL-IQ liver fat and liver iron measurements diverge from measurements obtained with conventional IOP and T2* mapping. To accomplish this task, we evaluate agreement between IDEAL-IQ and IOP methods, as well as the inter-reader agreement of these methods.

\section{Methods \\ Study population}

This retrospective study was performed after obtaining an institutional review board-approved waiver of informed consent. Of all patients that received a liver MRI at our institution between May 2016 and June 2017 and who underwent at least 1 cycle of chemotherapy, 54 consecutive patients were identified whose MRI studies included: [1] T1-in-and-out-of-phase, [2] IDEAL-IQ and [3] T2* mapping. Patient age ranged from 5 to $76($ mean $=36.7)$. There were 32 males and 22 females.

\section{MRI imaging parameters}

All patients were scanned on a $1.5 \mathrm{~T}$ MRI (OptimaMR450w, GE Healthcare) with a 32 Channel Torso Array Coil. The IDEAL IQ sequence had the following parameters: TR 10; TE 4.7; number of echoes 6, ranging from $1.1 \mathrm{~ms}$ to $6.38 \mathrm{~ms}$ FOV $35-40 \mathrm{~cm}$; matrix size $128 \times 128$; pixel bandwidth $325 \mathrm{~Hz}$; flip angle 6; slice thickness $10 \mathrm{~mm}$; space between slices $5 \mathrm{~mm}$. The scan was acquired during a single breath hold, lasting less than $30 \mathrm{~s}$. The IOP sequence had the following parameters: single breath hold acquisition lasting $15 \mathrm{~s}$, TR: $150 \mathrm{~ms}$, out-of-phase TE $2.115 \mathrm{~ms}$, in-phase TE: 4.252, FOV: $35-40 \mathrm{~cm}$, matrix $320 \times 192$, slice thickness $7 \mathrm{~mm}$, space between slices $1 \mathrm{~mm}$.

Post-processing software, provided by the manufacturer, was used to generate fat fraction maps and T2\% R2* maps [28].

\section{Image post-processing}

Using a dedicated workstation (Centricity PACS, RA1000 Workstation and Exam Manager, GE Healthcare), two radiologists independently reviewed all patient cases. For each case, the radiologists independently placed three circular regions of interest (ROIs) in the liver, and three circular ROIs in the spleen on the IOP images. To minimize fluctuations due to coil sensitivity, all ROIs were drawn within a $5 \mathrm{~cm}$ deep circular band extending from 10 to $15 \mathrm{~cm}$ from the bore isocenter (Fig. 1). ROIs included 100-300 voxels. The three ROIs in the liver were placed on a plane passing through the main portal vein division: one in the right lobe of the liver, one in segment 4 of the liver and one in segment $2 / 3$ of the liver, as demonstratively shown in Fig. 1. All ROIs were placed in the liver avoiding major vessels, ligaments and bile ducts, making sure that each 


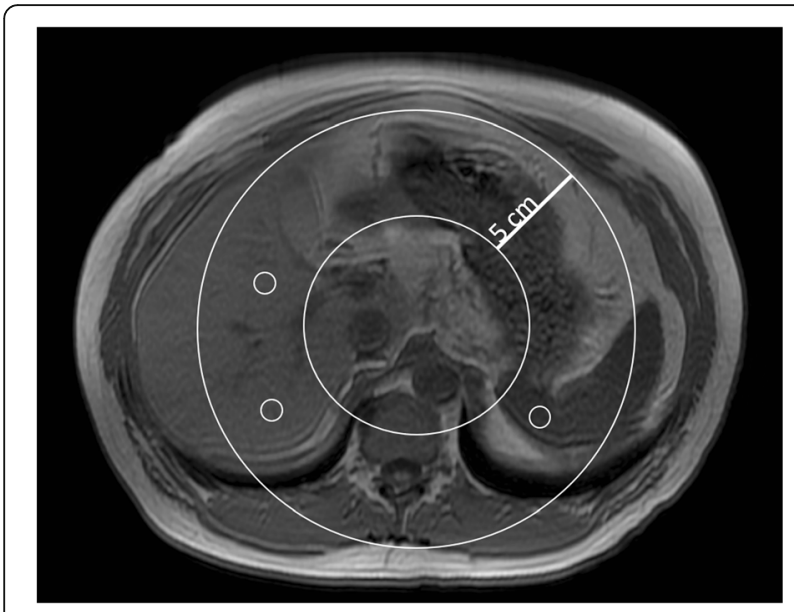

Fig. $1 \mathrm{ROI}$ placement. A $5 \mathrm{~cm}$ thick band was placed around the bore isocenter. Readers were instructed to place 3 ROIs in the liver and 3 in the spleen, on a plane passing through the portal vein division. Readers were instructed to place the ROls as close as possible to the midpoint of the $5 \mathrm{~cm}$ band, when possible, trying to avoid vessels and bile ducts

ROI was surrounded by liver parenchyma. ROIs were placed in the same positions on the fat fraction maps and $\mathrm{R}^{*}$ maps. We used the median values for all measured ROIs, in accordance with prior work [29]. Spleen ROIs were placed only on IP and OP images, along the same 5-cm band.

\section{Fat fraction, conventional method}

The ROIs described above were used to calculate fat fraction with the following "conventional method" formula [12]:

$$
\begin{aligned}
& \text { Liver fat fraction } \%= \\
& \qquad \frac{\frac{\text { SI InPhase }_{\text {liver }}}{\text { SI InPhase }_{\text {spleen }}}-\frac{\text { SI OutofPhase }_{\text {liver }}}{\text { SI OutofPhase }_{\text {spleen }}}}{2 * \frac{\text { SI InPhase }_{\text {liver }}}{\text { SI InPhase }_{\text {spleen }}}}
\end{aligned}
$$

In three patients, the spleen intensity was markedly decreased, presumably secondary to iron overload. In these patients, ROIs were instead placed in the paraspinal muscles, a less ideal choice given their smaller size and susceptibility to partial volume effects.

\section{Fat fraction, IDEAL-IQ method}

The liver ROIs described above were placed on the IDEAL-IQ fat fraction reconstruction to generate fat estimates.

\section{Iron quantification, conventional method}

The multiecho gradient echo acquisition was processed with GE software, which used three liver ROIs to generate $\mathrm{T} 2 * / \mathrm{R} 2 *$ value estimates for each patient. R2* value cutoffs were then employed to assign patients into the following categories: normal liver iron, mildly elevated liver iron, moderately elevated liver iron, severely elevated liver iron. There is a linear correlation between R2* values and liver-iron-concentration (LIC). Since R2* values are directly proportional to iron concentration, they were used as a primary measurement related to intrahepatic iron [29-31]. Increased intrahepatic iron levels were based on an $\mathrm{R} 2 *$ cutoff value that was arbitrary and approximately considered above $71 \mathrm{~s}^{-1}$ based on previous literature, since the only conversion software, approved and commercially available is a propertary software which was not available at the time of the study [30].

\section{Statistical analysis}

Intraclass correlation coefficient (ICC) was estimated to evaluate agreement on iron level and fat fraction between conventional MRI and IDEAL, as well as between two readers. Weighted kappa statistic with squared weights was used to assess agreement between iron level groups (normal: $<71 \mathrm{~s}^{-1}$; mild: $71-270 \mathrm{~s}^{-1}$; moderate: 270-588 $\mathrm{s}^{-1}$; and severe: $>588 \mathrm{~s}^{-1}$ ) based on conventional MRI measurement and IDEAL measurement as well as between readers [30, 32]. Exact Wilcoxon signed rank test was used to compare iron level and fat fraction between conventional MRI and IDEAL, in all patients, patients with normal iron level, and patients with above normal iron level.

Kappa values were interpreted as follows: $0.00-0.20$, slight agreement; $0.21-0.40$, fair agreement; 0.41-0.60, moderate agreement; 0.61-0.80, substantial agreement; and 0.81-1.00, almost perfect agreement. A test with $p$-value $<0.05$ was considered statistically significant. No adjustment on multiple testing was applied considering hypothesis generating purpose of the study. All statistical analyses were performed in software packages $R$ version 3.3 (The $\mathrm{R}$ Foundation for Statistical Computing, Vienna, Austria).

\section{Results}

Three patients were excluded because IDEAL-IQ reconstruction failed due to respiratory motion artifact. One patient was excluded because the T2* mapping GE software failed. The final study population consisted of 50 patients (30 males, 20 females, 50-78 years old).

In the literature, proposed PDFF intervals for each histological steatosis grade in nonalcoholic fatty liver disease are: $0-6.4 \%$ for grade 0 (normal); $6.5-17.4 \%$ for grade 1 (mild); $17.5-22.1 \%$ for grade 2 (moderate); and $22.2 \%$ or greater for grade 3 (severe) [19, 33]. Using IDEAL-IQ fat fraction quantification, 12 of 50 patients (26\%) had moderate or severe hepatic steatosis and 38 of 
50 patients (74\%) had normal liver fat or mild hepatic steatosis.

In the literature, $71 \mathrm{~s}^{-1}$ is generally taken as the normal R2* value cutoff $[34,35]$. Using IDEAL-IQ quantification, 37 of $50(74 \%)$ patients had abnormal liver iron level, and 13 of $50(26 \%)$ patients had low/normal liver iron levels [30, 32].

Ten patients (20\%) had both elevated iron and moderate/severe liver fat (Fig. 2). Table 1 summarizes the iron and fat measurements for all methods in both readers.

Across all patients, there was almost perfect agreement between readers for IDEAL fat fraction $(\mathrm{ICC}=0.957$ ) and IDEAL R2* (ICC = 0.971) measurements, but only substantial agreement between readers for conventional fat fraction measurements $(\mathrm{ICC}=0.626$ ). Conventional iron measurements were generated automatically on GE software and thus were not subject to inter-reader agreement analysis.

For both readers, there was poor intra-reader agreement $(\mathrm{ICC}=0.026, \mathrm{ICC}=0.028)$ between conventional fat measurements and IDEAL-IQ fat measurements. For both readers, there was fair intra-reader agreement $(\mathrm{ICC}=0.253, \mathrm{ICC}=0.291$ ) between conventional iron measurements and IDEAL-IQ iron measurements (Table 2).

Across all patients, the fat fractions calculated with IOP were statistically significantly different from those calculated with IDEAL-IQ (reader 1: $p<0.001$, reader 2: $p<0.001$ ). When only including patients with above normal iron level $(n=37)$, for both readers, the fat fractions calculated with IOP were statistically significantly different from those calculated with IDEAL-IQ (reader 1: $p<0.001$, reader 2: $p<0.001)$. However, when only including patients with low/normal iron levels $(n=13)$, the fat fractions calculated with IOP were not clearly statistically significantly different from those calculated with IDEAL-IQ $(p=0.068, p=0.048)$. See Table 3.

\section{Discussion}

In this paper, we demonstrate that a substantial number (20\%) of cancer patients undergoing liver MRI at our institution have both elevated liver fat and elevated liver iron. IDEAL-IQ, a confounder-corrected method to measure both liver fat and liver iron, is an important tool for early detection of hepatic steatosis in this patient population.

We show almost perfect inter-reader agreement for both fat measurements using IDEAL-IQ, far better than the conventional methods. This is an important point for clinicians who wish to follow a patient's hepatic steatosis over time.

Furthermore, we show that there is poor agreement between IOP and IDEAL-IQ fat measurements. We show that the difference is very pronounced in patients with concomitant iron $(p<0.001, p<0.001)$, but not univocally statistically significant in patients with normal levels of iron $(p=0.068, p=0.048)$. This suggests that differences in fat fraction calculation

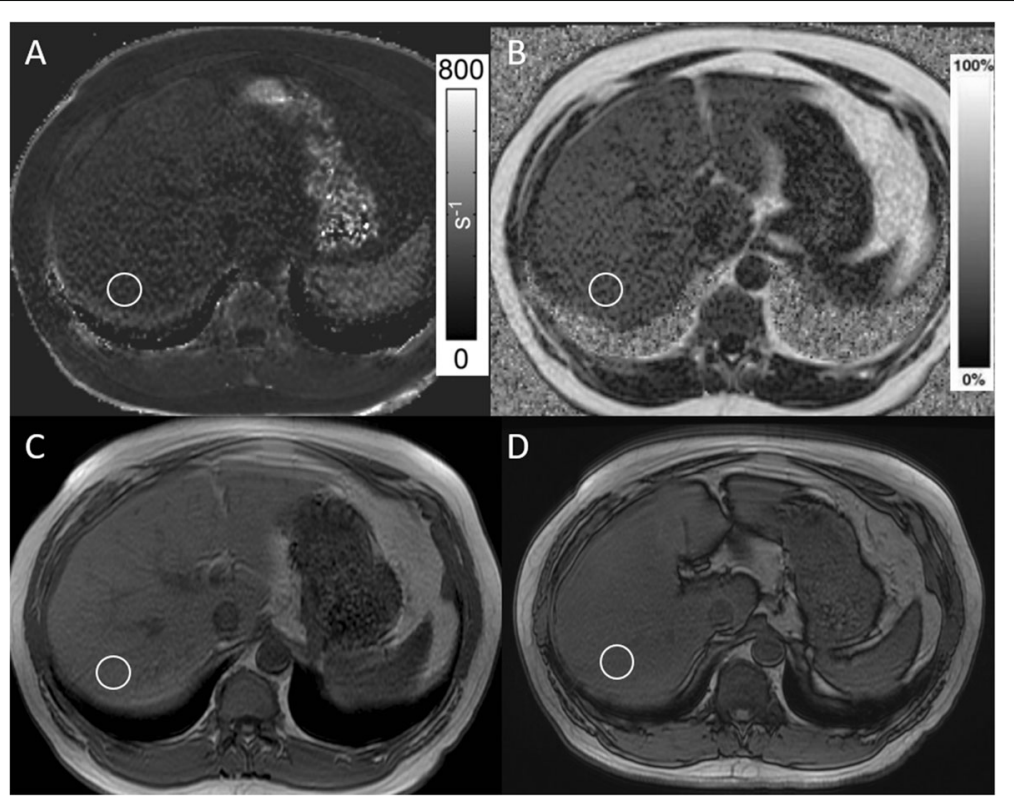

Fig. 2 Patient with mild iron overload. a R2* map with ROI demonstrating R2* value of $200 \mathrm{~s}^{-1}$. b Fat fraction map with ROI demonstrating $25 \%$ fat fraction. $\mathbf{c}$ In-phase and $\mathbf{d}$ Out-of-phase images demonstrate no signal drop-out within the ROI, demonstrating how elevated liver iron and elevated liver fat cancel on conventional methods and lead to underestimation of both liver fat and liver iron 
Table 1 Summary of R2* iron measurement and fat fraction in reader 1 and 2

\begin{tabular}{lll}
\hline & Median (Range) & Mean \pm SD \\
\hline R2* iron level conventional MRI & $109.2(31.3,714.3)$ & $126.8 \pm 109.6$ \\
R2* iron level IDEAL, reader 1 & $169(33,722.2)$ & $194.3 \pm 149.5$ \\
R2* iron level IDEAL, reader 2 & $161.8(32.5,709.3)$ & $188 \pm 140.6$ \\
Fat fraction IDEAL, reader 1 & $11.4(1,93.8)$ & $16.3 \pm 19.5$ \\
Fat fraction IDEAL, reader 2 & $11.1(1.2,99.7)$ & $17.6 \pm 21.4$ \\
Fat fraction conventional MRI (1), reader 1 & $1.3(-205.5,35)$ & $-7.4 \pm 41.4$ \\
Fat fraction conventional MRI (1), reader 2 & $1.3(-148.5,36.6)$ & $-6.8 \pm 35.6$ \\
Fat fraction conventional MRI (2), reader 1 & $1.7(-211.6,34.9)$ & $-6.4 \pm 40.6$ \\
Fat fraction conventional MRI (2), reader 2 & $1.8(-167.3,39)$ & $-6.6 \pm 37.6$ \\
\hline
\end{tabular}

between the IOP and IDEAL-IQ methods are more pronounced at higher iron levels than at lower iron levels. IDEAL-IQ has been previously validated with liver biospies and spectroscopic data and is the gold standard of this study, and so this difference is interpreted as the failure of IOP to measure fat in the presence of iron [36-39]. These results highlight that IOP is an inadequate technique to evaluate for liver fat in cancer patients, who are at high risk of elevated liver iron.

Small sample size did not permit us statistically analyze whether IDEAL-IQ fat fraction measurements were statistically significantly higher than IOP fat measurements. However, as our summary tables show, both the mean and the median of the IDEAL-IQ estimates for both fat and iron were higher than the mean and median conventional method estimates, for all readers. Further evaluation of this trend is warranted, since it suggests that, in the presence of both fat and iron, IDEAL-IQ is more sensitive detector of fat, permitting earlier detection of hepatic steatosis.

Table 2 Agreement on $\mathrm{R}^{*}$ iron level and fat fraction, between conventional MRI and IDEAL, as well as between readers

\begin{tabular}{llll}
\hline & ICC & Lower 95\%Cl & Upper 95\%Cl \\
\hline Between conventional MRI and IDEAL & & \\
R2* Iron level, Reader1 & 0.253 & -0.025 & 0.494 \\
R2* Iron level, Reader2 & 0.291 & 0.016 & 0.524 \\
Fat fraction, Reader1 & 0.026 & -0.252 & 0.300 \\
Fat fraction, Reader2 & 0.028 & -0.249 & 0.302 \\
Between readers & & & \\
IDEAL R2* iron & 0.971 & 0.950 & 0.984 \\
IDEAL fat fraction & 0.957 & 0.925 & 0.975 \\
Conventional fat fraction & 0.626 & 0.422 & 0.770 \\
\hline
\end{tabular}

Agreement on R2* iron level and fat fraction between conventional MRI and IDEAL was poor. Using IDEAL, the agreement was high between readers on both R2* iron level and fat fraction. On conventional MRI, the reader agreement was moderate on fat fraction
The major limitation of this study is the lack of a pathological gold standard; we do not have liver biopsy information for our study population. However, IDEAL-IQ has been pathologically validated in several prior studies [36-39]. As such, it is reasonable to consider IDEAL-IQ the best standard of reference for this study.

\section{Conclusion}

Cancer patients on chemotherapy are at increased risk for concomitantly elevated liver iron and elevated liver fat $[4,6,40]$. We found that $20 \%$ of cancer patients undergoing liver MRI with IDEAL-IQ and T2* mapping at our institution demonstrated both elevated liver iron and moderate/severe liver fat. We show that fat measurements using IDEAL-IQ and IOP diverge in patients with concomitantly elevated liver fat and liver iron. Given prior work validating IDEAL-IQ, these diverging measurements indicates that IOP is inadequate to screen for hepatic steatosis in our cancer population IDEAL-IQ demonstrated good inter-reader agreement for all liver iron and liver fat measurements.

Table 3 R2* iron level and fat fraction between conventional MRI and IDEAL

\begin{tabular}{llll}
\hline & Conventional MRI & IDEAL & $p$ Value \\
\hline All patients & & & \\
Fat fraction, reader 1 & $1.3(-205.5,35)$ & $11.4(1,93.8)$ & $<0.001$ \\
Fat fraction, reader 2 & $1.3(-148.5,36.6)$ & $11.1(1.2,99.7)$ & $<0.001$ \\
Patients with above normal R2* iron level $(n=37)$ & \\
Fat fraction, reader 1 & $1.8(-121.7,32)$ & $12.3(1,93.8)$ & $<0.001$ \\
Fat fraction, reader 2 & $0.6(-148.5,32.7)$ & $11.9(1.2,99.7)$ & $<0.001$ \\
Patients with low/normal R2* iron level $(n=13)$. & \\
Fat fraction, reader 1 & $1(-205.5,35)$ & $4.5(1.9,52.3)$ & 0.068 \\
Fat fraction, reader 2 & $3.2(-115.6,36.6)$ & $10.3(2.2,60)$ & 0.048 \\
\hline
\end{tabular}




\section{Abbreviations}

ICC: Intraclass correlation coefficient; IDEAL-IQ: Iterative decomposition of water and fat with echo asymmetry and least squares estimation; IOP: In and out of phase; IP: In phase; MRI: Magnetic resonance imaging; OP: Out of phase; PDFF: Proton density fat fraction; ROI: Region of interest; SI: Signal intensity

\section{Acknowledgements}

The study was approved by the local IRB with a waiver for informed consents. The authors were equally involved in acquisition of data, analysis and interpretation of data, drafting of the manuscript, critical revision of the manuscript for important intellectual content, statistical analysis, technical, or material support of this study.

Grant support was provided by MSK Cancer Center Support Grant/Core Grant P30 CA008748.

Work by Giuseppe Corrias was partially supported by a scholarship awarded by ISSNAF Imaging Science Chapter.

\section{Funding}

Grant support was provided by MSK Cancer Center Support Grant/Core Grant P30 CA008748. Work by Giuseppe Corrias was partially supported by a scholarship awarded by ISSNAF Imaging Science Chapter.

\section{Availability of data and materials}

The datasets during and/or analysed during the current study available from the $\mathrm{C}$ orresponding author on reasonable request.

\section{Authors' contributions}

Each author contributed to the preparation of the manuscript as follows: GC, SE-K, SR, and LM, conceived and planned the study. GC, SK, MF, and SM planned and carried out the experiments. JZ, MC, SR, contributed to the statistical analysis and to the interpretation of the results. SE-K, GC, and LM took the lead in writing the manuscript. All authors provided critical feedback and helped shape the research, analysis and manuscript. Each author has participated sufficiently in this submission to take public responsibility for its content. All authors read and approved the final manuscript.

\section{Ethics approval and consent to participate}

Institutional Review Board approval was obtained. Written informed consent was waived by the Institutional Review Board.

\section{Consent for publication}

Not applicable

\section{Competing interests}

The authors declare that they have no competing interests.

\section{Publisher's Note}

Springer Nature remains neutral with regard to jurisdictional claims in published maps and institutional affiliations.

\section{Author details}

${ }^{1}$ Department of Radiology, Memorial Sloan Kettering Cancer Center, 1275 York Avenue, New York, NY 10065, USA. ²Department of Radiology, University of Cagliari, Via Università, 40, 09124 Cagliari, CA, Italy. ${ }^{3}$ Department of Statistics, Memorial Sloan Kettering Cancer Center, 1275 York Avenue, New York, NY 10065, USA. ${ }^{4}$ IRCCS SDN, Naples, Italy. ${ }^{5}$ Global MR Applications and Workflow, GE Healthcare, New York, NY, USA. ${ }^{6}$ Department of Radiology, University of Wisconsin-Madison, Madison, WI, USA. ${ }^{7} 300$ East 66th Street, New York, NY 10021, USA

Received: 15 May 2018 Accepted: 14 September 2018

Published online: 12 December 2018

\section{References}

1. Merkle EM, Nelson RC. Dual gradient-echo in-phase and opposed-phase hepatic MR imaging: a useful tool for evaluating more than fatty infiltration or fatty sparing. Radiographics. 2006;26(5):1409-18.

2. Westphalen AC, Qayyum A, Yeh BM, Merriman RB, Lee JA, Lamba A, et al. Liver fat: effect of hepatic iron deposition on evaluation with opposedphase MR imaging. Radiology. 2007;242(2):450-5.
3. Longo R, Pollesello P, Ricci C, Masutti F, Kvam BJ, Bercich L, et al. Proton MR spectroscopy in quantitative in vivo determination of fat content in human liver steatosis. J Magn Reson Imaging. 1995;5(3):281-5.

4. Vauthey JN, Pawlik TM, Ribero D, Wu TT, Zorzi D, Hoff PM, et al. Chemotherapy regimen predicts steatohepatitis and an increase in 90-day mortality after surgery for hepatic colorectal metastases. J Clin Oncol. 2006; 24(13):2065-72

5. Sharma A, Houshyar R, Bhosale P, Choi J-I, Gulati R, Lall C. Chemotherapy induced liver abnormalities: an imaging perspective. Clin Mol Hepatol. 2014;20(3):317.

6. Cho CS, Curran S, Schwartz LH, Kooby DA, Klimstra DS, Shia J, et al. Preoperative radiographic assessment of hepatic steatosis with histologic correlation. J Am Coll Surg. 2008;206(3):480-8.

7. Maor $Y$, Malnick S. Liver injury induced by anticancer chemotherapy and radiation therapy. Int J Hepatol. 2013:2013:815105.

8. Anderson L, Holden S, Davis B, Prescott E, Charrier C, Bunce N, et al. Cardiovascular T2-star (T2*) magnetic resonance for the early diagnosis of myocardial iron overload. Eur Heart J. 2001;22(23):2171-9.

9. Yu H, McKenzie CA, Shimakawa A, Vu AT, Brau AC, Beatty PJ, et al. Multiecho reconstruction for simultaneous water-fat decomposition and $\mathrm{T2}^{*}$ estimation. J Magn Reson Imaging. 2007:26(4):1153-61.

10. Idilman IS, Gumruk F, Haliloglu M, Karcaaltincaba M. The feasibility of magnetic resonance imaging for quantification of liver, pancreas, spleen, vertebral bone marrow, and renal cortex $\mathrm{R}^{*}$ and proton density fat fraction in transfusion-related Iron overload. Turk J Haematol. 2016;33(1):21-7.

11. Horng DE, Hernando D, Reeder SB. Quantification of liver fat in the presence of iron overload. J Magn Reson Imaging. 2017:45(2):428-39.

12. Reeder SB, Cruite I, Hamilton G, Sirlin CB. Quantitative assessment of liver fat with magnetic resonance imaging and spectroscopy. J Magn Reson Imaging. 2011;34(4):729-49.

13. Campo CA, Hernando D, Schubert T, Bookwalter CA, Pay AJV, Reeder SB. Standardized approach for ROI-based measurements of proton density fat fraction and R2* in the liver. AJR Am J Roentgenol. 2017;209(3):592-603.

14. Hong CW, Fazeli Dehkordy S, Hooker JC, Hamilton G, Sirlin CB. Fat quantification in the abdomen. Top Magn Reson Imaging. 2017;26(6):221-7.

15. Reeder SB, Pineda AR, Wen Z, Shimakawa A, Yu H, Brittain JH, et al. Iterative decomposition of water and fat with echo asymmetry and least-squares estimation (IDEAL): application with fast spin-echo imaging. Magn Reson Med. 2005;54(3):636-44.

16. Reeder SB, McKenzie CA, Pineda AR, Yu H, Shimakawa A, Brau AC, et al. Water-fat separation with IDEAL gradient-echo imaging. J Magn Reson Imaging. 2007;25(3):644-52.

17. Kang GH, Cruite I, Shiehmorteza M, Wolfson T, Gamst AC, Hamilton G, et al. Reproducibility of MRI-determined proton density fat fraction across two different MR scanner platforms. J Magn Reson Imaging. 2011;34(4):928-34.

18. Johnson BL, Schroeder ME, Wolfson T, Gamst AC, Hamilton G, Shiehmorteza $M$, et al. Effect of flip angle on the accuracy and repeatability of hepatic proton density fat fraction estimation by complex data-based, $\mathrm{T} 1$ independent, T2*-corrected, spectrum-modeled MRI. J Magn Reson Imaging. 2014;39(2):440-7.

19. Tang A, Desai A, Hamilton G, Wolfson T, Gamst A, Lam J, et al. Accuracy of MR imaging-estimated proton density fat fraction for classification of dichotomized histologic steatosis grades in nonalcoholic fatty liver disease. Radiology. 2015;274(2):416-25.

20. Hamilton G, Middleton MS, Hooker JC, Haufe WM, Forbang NI, Allison MA, et al. In vivo breath-hold (1) H MRS simultaneous estimation of liver proton density fat fraction, and $\mathrm{T} 1$ and $\mathrm{T} 2$ of water and fat, with a multi-TR, multi-TE sequence. J Magn Reson Imaging. 2015;42(6):1538-43.

21. Kim KY, Song JS, Kannengiesser S, Han YM. Hepatic fat quantification using the proton density fat fraction (PDFF): utility of free-drawn-PDFF with a large coverage area. Radiol Med. 2015;120(12):1083-93.

22. Yu H, Shimakawa A, McKenzie CA, Brodsky E, Brittain JH, Reeder SB. Multiecho water-fat separation and simultaneous $R 2^{*}$ estimation with multifrequency fat spectrum modeling. Magn Reson Med. 2008;60(5):1122-34.

23. Ong ES, Chor CF, Zou L, Ong CN. A multi-analytical approach for metabolomic profiling of zebrafish (Danio rerio) livers. Mol BioSyst. 2009;5(3):288-98.

24. Karcaaltincaba M, Idilman I, Celik A. Focal sparing of iron and fat in liver tissue in patients with hemosiderosis: diagnosis with combination

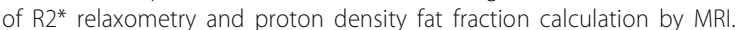
Diagn Interv Radiol. 2011;17(4):323-7. 
25. Chiang HJ, Lin LH, Li CW, Lin CC, Chiang HW, Huang TL, et al. Magnetic resonance fat quantification in living donor liver transplantation. Transplant Proc. 2014;46(3):666-8.

26. Idilman IS, Tuzun A, Savas B, Elhan AH, Celik A, Idilman R, et al. Quantification of liver, pancreas, kidney, and vertebral body MRI-PDFF in non-alcoholic fatty liver disease. Abdom Imaging. 2015;40(6):1512-9.

27. Serai SD, Dillman JR, Trout AT. Proton density fat fraction measurements at 1.5- and 3-t hepatic mr imaging: same-day agreement among readers and across two imager manufacturers. Radiology. 2017;284(1):244-54.

28. Wood JC. Magnetic resonance imaging measurement of iron overload. Curr Opin Hematol. 2007;14(3):183

29. Roberts NT, Hernando D, Holmes JH, Wiens CN, Reeder SB. Noise properties of proton density fat fraction estimated using chemical shift-encoded MRI. Magn Reson Med. 2018.

30. Virtanen JM, Komu ME, Parkkola RK. Quantitative liver iron measurement by magnetic resonance imaging: in vitro and in vivo assessment of the liver to muscle signal intensity and the R2* methods. Magn Reson Imaging. 2008; 26(8):1175-82

31. Pirasteh A, Yuan Q, Hernando D, Reeder SB, Pedrosa I, Yokoo T. Intermethod reproducibility of biexponential R2 MR relaxometry for estimation of liver iron concentration. Magn Reson Med. 2018. https://doi.org/10.1002/ mrm.27348. [Epub ahead of print].

32. Wood JC, Enriquez C, Ghugre N, Tyzka JM, Carson S, Nelson MD, et al. MRI R2 and R2* mapping accurately estimates hepatic iron concentration in transfusion-dependent thalassemia and sickle cell disease patients. Blood. 2005:106(4):1460-5.

33. Szczepaniak LS, Nurenberg P, Leonard D, Browning JD, Reingold JS, Grundy $S$, et al. Magnetic resonance spectroscopy to measure hepatic triglyceride content: prevalence of hepatic steatosis in the general population. Am J Physiol Endocrinol Metab. 2005;288(2):E462-8.

34. Henninger B, Kremser C, Rauch S, Eder R, Zoller H, Finkenstedt A, et al. Evaluation of MR imaging with $\mathrm{T} 1$ and $\mathrm{T} 2 *$ mapping for the determination of hepatic iron overload. Eur Radiol. 2012;22(11):2478-86.

35. St Pierre TG, Clark PR, Chua-Anusorn W, Fleming AJ, Jeffrey GP, Olynyk JK, et al. Noninvasive measurement and imaging of liver iron concentrations using proton magnetic resonance. Blood. 2005;105(2):855-61.

36. Hernando D, Kramer JH, Reeder SB. Multipeak fat-corrected complex 12*relaxometry: theory, optimization, and clinical validation. Magn Reson Med. 2013;70(5):1319-31.

37. Hernando D, Sharma SD, Ghasabeh MA, Alvis BD, Arora SS, Hamilton G, et al. Multisite, multivendor validation of the accuracy and reproducibility of proton-density fat-fraction quantification at 1.5T and $3 \mathrm{~T}$ using a fat-water phantom. Magn Reson Med. 2017;77(4):1516-24.

38. Bannas $\mathrm{P}$, Kramer $\mathrm{H}$, Hernando D, Agni R, Cunningham AM, Mandal $R$, et al. Quantitative magnetic resonance imaging of hepatic steatosis: validation in ex vivo human livers. Hepatology. 2015;62(5):1444-55.

39. Hines CDG, Agni R, Roen C, Rowland I, Hernando D, Bultman E, et al. Validation of MRI biomarkers of hepatic steatosis in the presence of iron overload in the Ob/Ob mouse. J Magn Reson Imaging. 2012;35(4):844-51.

40. Reeder SB, Sirlin CB. Quantification of liver fat with magnetic resonance imaging. Magn Reson Imaging Clin N Am. 2010;18(3):337-57 ix.

Ready to submit your research? Choose BMC and benefit from:

- fast, convenient online submission

- thorough peer review by experienced researchers in your field

- rapid publication on acceptance

- support for research data, including large and complex data types

- gold Open Access which fosters wider collaboration and increased citations

- maximum visibility for your research: over $100 \mathrm{M}$ website views per year

At BMC, research is always in progress.

Learn more biomedcentral.com/submissions 